\title{
GÉNERO Y ETNICIDAD: APORTES DESDE UNA ANTROPOLOGÍA JURÍDICA CRÍTICA
}

\author{
María Teresa Sierra
}

E $\mathrm{n}$ este texto me propongo abordar la relación entre el género y la etnicidad desde la perspectiva de una antropología jurídica crítica interesada en cuestionar visiones homogéneas y ahistóricas del derecho yla cultura. Desde esta perspectiva me interesa aportar al estudio del derecho en contextos interétnicos, refiriéndome a una experiencia colectiva de investigación que hemos tenido en distintas regiones indígenas del país en donde pretendimos dar cuenta de la manera en que el derecho (consuetudinario y estatal) construye y reproduce roles de género, así como las alternativas legales a disposición de las mujeres para resolver sus controversias (Sierra, en prensa). El objetivo último es poder relacionar lo que observamos, desde la mirada antropológica, de las prácticas sociales en la dimensión política de las reivindicaciones de las mujeres indígenas.

La perspectiva de género en el estudio antropológico del derecho y los procesos étnicos constituye un referente central para documentar la heterogeneidad constitutiva de las relaciones sociales y para dar cuenta de los diferenciales de poder que estructuran a todo derecho, incluido el derecho indígena. Este punto de vista cuestiona discursos relativistas que han tendido a construir visiones homogéneas y armónicas sobre las sociedades indígenas María Teresa Sierra, CIESAS-México. y sus sistemas normativos. Elaboraciones recientes que relacionan la problemática del género, la etnicidad y el derecho, muestran que dichos conceptos son construcciones culturales que deben ser analizados desde contextos históricos particulares para entender cómo las sociedades han construido la relación entre los sexos, cómo estas relaciones tienden a ser instituidas y legitimadas a través de normas y valores, y en qué medida la pertenencia étnica media esa relación. Se trata efectivamente de categorías históricas que cristalizan procesos sociales y relaciones de poder; en este sentido permiten cuestionar visiones que tienden a naturalizar las relaciones sociales impidiendo dar cuenta de las dinámicas de cambio y transformación en las que éstas se insertan. En las sociedades indígenas los sistemas normativos y los sistemas sexo-género ${ }^{1}$ no son autocontenidos ni ahistóricos sino que se definen en la intersección de normatividades y valores provenientes de la sociedad hegemónica regional. Esto no niega que podamos hablar de sistemas normativos, que responden a lógicas culturales propias, pero con la condición de entender que no son entidades petrificadas en el tiempo y fuera de la historia, como suelen construirlos algunas visiones ideologizadas en torno al derecho indígena o el mismo derecho positivo estatal. Asimismo resulta de fundamental importancia comprender que el género es 
un constructor cultural e histórico, lo que significa que las relaciones entre los sexos pueden modificarse, por lo que no siempre han sido las mismas. En el caso de las comunidades indígenas el trabajo antropológico debe mostrar cómo se manifiestan y reproducen los roles genéricos en distintos contextos culturales, cómo se han ido transformando y el papel que juegan los referentes normativos (la ley y la costumbre) en su reproducción y en los juegos del poder.

\section{Usos del derecho y relaciones de género en regiones indígenas}

En los siguiente renglones, me refiero a una investigación colectiva que realizamos en diferentes regiones indígenas en donde buscamos dar cuenta, entre otros temas, de las maneras en que las mujeres indígenas se confrontan con el derecho, tanto en el espacio de sus comunidades cómo en las instancias del Estado, y como recurren a distintos referentes de legalidad para hacer frente a sus problemáticas (Sierra, op.cit.) La confrontación con la justicia resulta ser un espacio revelador para dar cuenta de las normas, valores y roles de género que legitiman las distintas sociedades, ya que al dirimir las controversias ante las autoridades o mediadores tradicionales, se ponen en juego discursos sobre el deber ser genérico, así como las conflictividades y relaciones de poder que atraviesa la relación entre los sexos.

La investigación abarcó varias regiones indígenas lo cual nos ha permitido tener una visión amplia sobre la problemática de las mujeres indígenas ante la ley: así tenemos información de mujeres totonacas de la Sierra de Papantla (Victoria Chenaut), de mujeres mixtecas de Guerrero (Maribel Nicasio), de mujeres nahuas de la Sierra norte de Puebla en Cuetzalan (Ivette Vallejo y María Teresa Sierra) y Huauchinango (María Teresa Sierra), de mujeres tsotsiles de Chiapas (Jane Collier), de mujeres mixes en Oaxaca (Elisa Cruz), y de mujeres otomíes y mazahuas en la Ciudad de México (Rebecca Igreja). ${ }^{2}$
Un primer análisis comparativo de los procesos que observamos en estas regiones nos permite constatar el alto nivel de litigio que se da en las instancias de la justicia indígena en los cuales participan mujeres, así como las estrategias que desarrollan para resolver sus controversias. Muchos de los asuntos que llevan las mujeres ante sus autoridades se refieren a problemas que involucran relaciones genéricas y en gran medida revelan tensiones al interior de los grupos domésticos como sucede con los conflictos entre esposos, entre mujer y suegra, entre mujeres, y entre grupos familiares en torno a problemas de noviazgo, por ejemplo. No es mi intención referirme aquí a los distintos asuntos y su particularidad de acuerdo con las diferentes regiones, pero sí destacar que las mujeres ven en la justicia local un espacio para dirimir sus asuntos. De hecho según hemos podido constatar en diferentes regiones la jurisdicción indígena sigue siendo la instancia principal de resolución de disputas en las comunidades. Otro elemento que salta a la vista es la alta conflictividad existente en el interior de los grupos domésticos y que, en gran medida, son las mujeres quienes en diferentes momentos del ciclo doméstico se ven afectadas por relaciones de subordinación que suelen implicar violencia física y verbal. En muchos casos, sobre todo durante conflictos matrimoniales, sale a relucir la concepción según la cual el hombre tiene la prerrogativa de corregir a su mujer y de llegar incluso al maltrato si no cumple con sus obligaciones domésticas, discurso que suele ser enarbolado por los hombres para justificar su actuación; las mujeres por su parte suelen quejarse de que el hombre es irresponsable y no ofrece lo necesario para el gasto familiar, se emborracha o anda con otra mujer (Vallejo, 2000). La observación y análisis de diferentes juicios en las comunidades nos ha permitido reconstruir los argumentos y referentes normativos que las autoridades indígenas valoran durante los litigios para hacer ver las obligaciones de cada pareja y la necesidad de que se ajusten a los roles estipulados. Generalmente las conciliaciones buscan llegar a acuerdos negociados, no 
necesariamente a soluciones justas, con el fin de recomponer las relaciones familiares, y buscar que las partes reconsideren su comportamiento, según hemos podido documentar entre los nahuas, como entre los zinacantecos ( $c f r:$ Collier, 1995). En este sentido no se cuestiona el hecho mismo de la violencia sino el incumplimiento de los roles. Sin embargo, las mujeres no son sólo víctimas de la violencia o de situaciones que las subordinan; en ocasiones ellas mismas reproducen comportamientos violentos hacia sus maridos, pero sobre todo hacia otras mujeres, especialmente cuando éstas se meten con sus hombres. Un número considerable de asuntos de este tipo suelen llegar ante los jueces de las comunidades como pudimos constatar en Cuetzalan, y en Huauchinango (Sierra 1995). En otros casos las mujeres son vistas como transgresoras de las normas y por lo mismo son criticadas y en ocasiones presionadas a aclarar comentarios y calumnias (Vallejo op. cit). En algunas regiones la práctica de la poliginia es aceptada, lo que lleva a que las autoridades locales arreglen asuntos que involucran a las dos esposas, sin necesariamente cuestionar el hecho mismo de la poliginia (Chenaut, 1999; Nicasio 2001).

Llama la atención el alto nivel de conflictividad sexual involucrado en los litigios en la región de Cuetzalan, afectando particularmente a las mujeres, y en ocasiones a los niños, lo que contrasta con lo que hemos observado en otras regiones como sucede entre los nahuas de Huauchinango, Puebla, en donde el nivel de violencia doméstica es menor, pero es mayor también el control sobre las mujeres. Algo similar sucede entre los zinacantecos de Chiapas. Según documenta Collier (en prensa), las reconciliaciones familiares del grupo doméstico tienden a que se reestablezca la relación familiar para lo cual las autoridades ponen en juego diferentes estrategias, incluido el hecho mismo de recurrir al derecho del Estado si un marido no quiere cumplir con sus obligaciones, o no quiere cambiar su comportamiento: tal es lo que sucede cuando los jueces amenazan al hombre con la obligación legal de dar una pensión alimenticia hacia su familia, lo que en muchos casos suele disuadir al hombre de separarse. Otra es la realidad en Metlatónoc, Guerrero, donde las mujeres mixtecas tienen pocas alternativas para confrontar sus diputas en los ámbitos locales, en lo cual ha incidido la crisis de las instituciones comunitarias ante la situación de violencia y alta marginación de la zona, y la falta misma de estado de derecho (Nicasio en prensa).

Es de notar también que en algunas regiones resulta ser una práctica común para las mujeres acudir a las instancias del Estado cuando en las locales no encuentran alternativas a la justicia, tal como observamos en la Sierra norte de Puebla (en Cuetzalan y en Huauchinango), y en la región totonaca de Coyutla, lo que sin embargo no parece suceder entre las mujeres zinacantecas de los Altos de Chiapas, o las mujeres mixtecas de Metlatónoc. Al parecer tal situación tiene que ver con el tipo de presencia y de legitimidad diferenciada que el Estado ha tenido en las distintas regiones, por lo cual no es posible hacer generalizaciones. Así, es un hecho recurrente que cuando los asuntos no se dirimen localmente, las mujeres y hombres nahuas de Cuetzalan, y en menor medida de Huauchinango, recurren a las instancias del Estado, sobre todo las municipales, para dirimir sus asuntos. Las instancias municipales insertas en las regiones indígenas a pesar de ser centros del poder caciquil, controlados por mestizos, son instancias de intermediación entre el Estado y las comunidades que suelen ser vistas como "más neutrales" por los y las indígenas de la región, lo que permite abrir espacios de negociación para dirimir asuntos. Hemos constatado cómo incluso en estos espacios de intermediación es posible hacer valer las costumbres y llegar a acuerdos con base en negociaciones que, en ocasiones, contradicen la legalidad del Estado, sobre todo cuando se trata de asuntos considerados de menor gravedad. El espacio que puede abrirse depende, sin embargo. de la disposición 
que tengan las autoridades judiciales para buscar una salida negociada a las disputas, evitando así que se carguen los procesos legales. En otras ocasiones lo que prevalece es el discurso de la ley que se impone sobre cualquier otra consideración, lo que dependerá de la gravedad del asunto (Sierra, en prensa b).

¿Por qué acuden las mujeres a las instancias estatales? Según hemos observado las mujeres acuden a dichas instancias, como una manera de presionar hacia acuerdos que no consiguieron en sus comunidades, como una forma de amenazar a sus cónyuges recurriendo al peso de la ley, o porque consideran que tendrán más oportunidad de alcanzar soluciones más neutrales, ya que en ocasiones las autoridades de los pueblos son compadres del marido o la familia del esposo. Pero acuden también cuando están decididas a llevar a cabo procesos judiciales para defender sus derechos. Si bien estos casos son los menores, observamos que recurrir a la ley abre alternativas a las mujeres para confrontar costumbres, normas y poderes dentro de las comunidades, lo que sin duda también significa debilitar a las autoridades tradicionales. No hay, sin embargo, garantía de que en los espacios de la justicia estatal se resuelvan las controversias conforme a derecho, ya que se entra al laberinto de la legalidad, la discriminación y la corrupción que domina en estos espacios, lo cual lleva a que muchos delitos queden impunes, como es el caso de las violaciones. ${ }^{3}$

En los últimos tiempos hemos visto que las mujeres indígenas también están recurriendo a niveles legales superiores del Estado, al distrito judicial con las autoridades de la Primera Instancia, para llevar adelante procesos judiciales. En estos niveles domina una mayor profesionalización de la ley, se impone el discurso jurídico estatal como único referente de legalidad, y se obliga a buscar el apoyo de abogados o defensores que sirvan de intermediarios y traductores de los procesos legales. Lo que llama la atención es el número importante de asuntos penales y civiles en los que se ven involucradas mujeres de las comunidades, muchos de los cuales tienen que ver con violaciones, lesiones, despojos, pero también los hay asuntos que se refieren a la legalización de escrituras, juicios por pensión alimenticia, reconocimiento de hijos, hasta divorcios. ¿Qué tipo de mujeres son las que llegan a estos espacios y cómo hacen para confrontar la justicia?. Según hemos observado, quien acude a este nivel de justicia se ve expuesto a realizar gastos para pagar costos de los traslados, el papeleo y en ocasiones los apoyos legales. Lo que llama la atención es que una mayoría de estas mujeres proviene de comunidades lejanas, algunas de ellas son monolingües y de muy escasos recursos, pero están decididas a llevar adelante una demanda. Tal es, por ejemplo, el caso de doña Antonia, una mujer nahua del municipio Zacapoaxtla, en Puebla, con un dominio muy limitado del español, analfabeta, quien debió vencer una serie de obstáculos económicos y familiares para conseguir finalmente la escritura de su terreno, ante las amenazas de sus vecinos que querían despojarla de él (cfr:: Sierra, 2000).

Detrás de cada proceso judicial se esconden dramas en donde el litigio es sólo un momento en la acumulación de anteriores disputas que revelan una particular indefensión de las mujeres en las relaciones familiares, domésticas, y comunales, cuestión en la cual interviene la legitimación de las costumbres. ${ }^{4}$

\section{Cambios sociales y su impacto en las costumbres y en los roles de género}

La referencia a problemas recurrentes permitirá ilustrar la conflictividad vigente entre los géneros así como la situación particular de indefensión y violencia que viven las mujeres. Destaco en particular la manera en que los cambios sociales van incidiendo en la transformación de las costumbres y en la redefinición de los roles genéricos, si bien no se observa un cambio radical en ellos. Me refiero en particular a asuntos que hemos podido seguir en comunidades nahuas de Cuetzalan y 
en Huauchinango pero que son también vigentes en las otras regiones de nuestro estudio.

Muchos de los cambios que están impactando los roles genéricos tienen que ver con transformaciones estructurales de carácter económico y político que están atravesando a las comunidades y redefiniendo sus formas de organización. Los cambios deben verse en el contexto de cada región considerando el tipo de relación que las comunidades han tenido con el Estado, así como la fuerza de las comunidades para generar procesos organizativos.

Un eje central de estos cambios ha sido la escuela ya que ha incidido en que los jóvenes se conozcan, posibilitando relaciones y una interacción que antes resultaba difícil para hombres y mujeres, lo cual repercute en el tipo de noviazgo que se desarrolla en las comunidades. Así, observamos que el noviazgo tradicional basado en la pedida de la novia a través de regalos (el llamado tlapalole entre los nahuas), que solía llevar a matrimonios arreglados, está siendo transformado radicalmente por nuevas prácticas de noviazgo que conllevan la decisión de los jóvenes de involucrarse en una relación y que suelen terminar muchas veces con la fuga de la novia, generalmente para vivir en casa de los padres del muchacho. ${ }^{5}$ Estas nuevas prácticas, sin embargo, generan conflictos, sobre todo si los padres de la muchacha no están conformes con la decisión de los muchachos, ya sea porque buscan recuperar parte de una dote, o porque consideran que su hija no está madura para casarse. Algunos de estos asuntos terminan ante las autoridades de las comunidades, y en general suelen resolverse con el compromiso de la familia del novio de cuidar y educar a la muchacha, y en ocasiones con un pago a la familia de la mujer que funciona como una especie de compensación (Cfr: Sierra, 1995). Los cambios en las costumbres del novia zgo no se dan de la misma manera en las distintas regiones y en ocasiones hay familias que continúan con la costumbre de la pedida y los rituales del matrimonio, particularmente si se tienen los recursos para financiar los festejos; también existen casos de mujeres, las de menos recursos, que se ven obligadas a casarse sin su voluntad o que aceptan ser la segunda mujer como una alternativa para que alguien las mantenga. Tal es el caso de Doña Antonia, de Zacapoaxtla, quien fue entregada sin su voluntad por sus padres a un señor mayor, siendo ella aún niña. Sin duda las opciones que tienen las mujeres ante el noviazgo y el matrimonio se relacionan con la posición económica de las familias, pero también con las opciones que les abren espacios como la escuela y la participación en organizaciones y en espacios públicos

La migración es también uno de los factores que más están afectando la organización local pues inciden en las costumbres y en la consecuente redefinición del lugar de las mujeres en los núcleos domésticos. En ocasiones las mujeres se ven obligadas a estar al frente del núcleo familiar, pero también muchas de ellas subordinadas a que el esposo migrante envíe su dinero, con frecuencia debe compartir con la suegra. Si bien las regiones estudiadas no son todavía centros de migración intensiva, estos procesos empiezan a darse. Puede verse, por ejemplo, cómo se generan nuevos conflictos en los grupos domésticos ante la ausencia del hombre y cómo las dinámicas de cambio afectan también la organización comunitaria, obligando a las mujeres a participar en las asambleas y a asumir cargos tradicionalmente ocupados por los hombres.

Las disputas por la tierra y por los bienes suelen estar detrás de una gran cantidad de asuntos que llegan a las instancias judiciales de las comunidades y del Estado. Dichos casos revelan la indefensión de las mujeres ante costumbres que no les reconocen el derecho a poseer bienes, sobre todo cuando muere el marido y la mujer queda viuda. Varios de los asuntos que pudimos documentar tienen que ver con conflictos al interior del grupo doméstico, de la viuda con familiares del esposo quienes han pretendido quitarle el terreno o la casa en la 
que vive porque consideran que no le pertenece o sostienen que ellos cuidarán las tierras de sus sobrinos. Si bien este tipo de conflictos nos son nuevos, ya que los hemos podido rastrear en las actas antiguas de conciliación en los juzgados de los pueblos, lo que sí es nuevo es la decisión de las mujeres de hacer uso de alternativas y lenguajes legales para defender sus derechos. Tal es, por ejemplo, lo que hemos visto cuando mujeres acuden hasta el distrito judicial y se ven involucradas en procesos judiciales para legalizar escrituras o disputar una herencia.

En los últimos tiempos han aumentado los juicios por el reconocimiento de nombre para los hijos y por la pensión alimenticia, en los cuales se ven involucradas mujeres indígenas. En ocasiones se trata de procesos que terminan en las instancias municipales, con el compromiso del hombre de reconocer al hijo o cumplir con la pensión; pero en otras ocasiones se siguen juicios para garantizar a las mujeres que su hijo sea reconocido por su padre, y de esa manera se le pueda forzar a que cumpla una pensión o más adelante a que el hijo tenga derecho a una herencia. Obviamente el recurrir a la justicia del Estado no garantiza el que se cumplan las sentencias y se ejerza la justicia. No obstante es indudable que la legalidad del Estado ha entrado a formar parte de los recursos jurídicos a disposición de mujeres y hombres para dirimir sus asuntos. Se trata, efectivamente, de nuevos procesos que expresan las dinámicas de cambio que se viven en las regiones indígenas, en las que intervienen de manera activa las organizaciones de defensoría en derechos humanos y derechos indígenas como veremos a continuación.

\section{Las organizaciones de derechos humanos y la reivindicación de las mujeres indígenas}

La presencia significativa de organismos de defensa de derechos humanos en algunas regiones indígenas del país así como de organizaciones de mujeres están jugando un papel importante en el campo de la justicia y particularmente en el acceso a la legalidad del Estado. Llama la atención la manera en que el lenguaje sobre los derechos y la legalidad está siendo utilizada en los asuntos que involucran a las mujeres y los hombres de las comunidades, lo que en ocasiones suele entrar en tensión con costumbres arraigadas en las comunidades; tal es lo que sucede cuando se reivindica el derecho a heredar en igualdad de condiciones, o se exige la pensión alimenticia, las actas de nacimiento para confirmar la filiación, el papel relevante de sellos y legalizaciones que se introducen como referentes de negociación en las jurisdicciones indígenas, cuestiones a las cuales están apelando las mujeres. El proceso se da de manera diferenciada y hay regiones en donde la presencia más significativa de ONGs, de instancias estatales de derechos humanos y otras instituciones del Estado como el DIF y el INI, ha propiciado que se recurra a argumentos legales y a las autoridades mestizas del Estado. Según pudimos constatar en nuestra investigación, dichos procesos organizativos y de promoción de defensoría jurídica son notorios en la región nahua de Cuetzalan, en municipios mixtecos de la montaña de Guerrero, y los Altos de Chiapas y en menor medida en la zona totonaca de Coyutla y en los municipios nahuas de la zona de Huauchinango. Por su parte las organizaciones de mujeres indígenas y mestizas, están propiciando una discusión importante sobre la problemática del género y los derechos indígenas, la que en lugares como Cuetzalan y San Cristóbal de las Casas está generando nuevas alternativas de defensa y motivando una reflexión sobre sus derechos y sus costumbres. Estos procesos están abriendo opciones a las mujeres para verbalizar sus problemáticas y en la medida de lo posible redefinir sus relaciones de género. En algunas regiones se han abierto también espacios de apoyo y defensa legal que facilitan a muchas su acceso a la jurisdicción del Estado, sin que ello signifique que se les garantice la justicia. Se 
observa también que estas organizaciones, específicamente las vinculadas con la defensoría en derechos humanos, tienden a convertirse en instancias alternativas de resolución de conflictos, consiguiendo dirimir una buena cantidad de asuntos que les llevan los miembros de las comunidades, con lo que evitan que muchos de ellos entren a los laberintos de la legalidad estatal. Sin duda tales organizaciones están abriendo nuevas opciones para confrontar diferencias y conflictos en el ámbito de las comunidades y ante las instancias del Estado.

Pero, como consecuencia de dichos procesos en los que se ven inmersas estas organizaciones, surgen contradicciones, sobre todo por el impacto de trasladar de manera "mecánica" el discurso de los derechos humanos al ámbito de las comunidades indígenas, sin considerar las especificidades culturales que determinan el ejercicio de las costumbres, lo cual ha propiciado el debilitamiento de la autoridad tradicional. Algunas organizaciones están abiertas a discutir estos temas y a pensar en alternativas para la defensa de los derechos humanos sin que conlleven la simple imposición de un discurso individualista, defensor de las garantías individuales. El reto es, entonces, construir propuestas que cuestionen poderes instituidos y visiones homogéneas del derecho para abrir opciones que desde los propios marcos culturales de la justicia indígena resulten liberadoras. Estamos efectivamente ante uno de los retos que involucra el debate actual sobre el reconocimiento de los derechos indígenas.

\section{Antropología jurídica y reclamos legales}

La visión antropológica de las prácticas jurídicas permite constatar la problemática cotidiana que viven las mujeres indígenas cuando se confrontan a la justicia tanto en las instancias comunitarias como en las estatales, cuestión que muestra el alto grado de conflictividad que caracteriza la relación entre los géneros, pero también el que en algunas regiones las mujeres están acudiendo a las instancias estatales y utilizando el lenguaje del derecho para buscar alternativas a sus demandas. Si bien recurrir a la legalidad del Estado no garantiza la aplicación de la ley y la justicia, y en muchas ocasiones los asuntos quedan impunes, también es cierto que el discurso de la legalidad está siendo visto por las mujeres indígenas como un referente de legitimidad para confrontar poderes locales y tradiciones instituidas. Al mismo tiempo el lenguaje del derecho está siendo utilizado en el ámbito mismo de las comunidades indígenas y se están abriendo instancias alternativas de resolución de disputas como es el caso de las organizaciones defensoras de derechos humanos que incorporan las demandas de las mujeres. Estos procesos repercuten en la práctica misma de la autoridad indígena, pues propician la vigilancia de los procesos legales por parte de las organizaciones, y discusiones en torno a ciertas costumbres. Observamos así que las jurisdicciones indígenas están siendo transformadas al incorporar en su seno nuevos lenguajes eincluso nuevos agentes de mediación, lo que trae consigo procesos contradictorios; así como esto puede enriquecer las dinámicas locales y la misma práctica de la autoridad, puede también significar que la autoridad indígena pierda legitimidad cuando una gran parte de los asuntos salen de las comunidades, y cuando dicha autoridad es vista como auxiliar a las autoridades del Estado. Uno de los principales retos que enfrentan las comunidades indígenas y sus autoridades es justamente el fortalecer sus jurisdicciones, para ser capaces de adecuar y transformar algunos marcos legales y referentes normativos y generar alternativas de justicia en el ámbito de las comunidades y regiones indígenas. Es decir, que permitan adecuar y dar salida a las demandas de los grupos subordinados, como sucede con las mujeres y con otras disidencias locales. Son las propias dinámicas sociales y económicas las que están propiciando cambios en la organización comunitaria y obligando a redefinir costumbres consideradas como difíciles de cambiar. Los 
cambios, sin embargo, no se corresponden con ideologías arraigadas que sustentan los sistemas sexogénero que suelen reproducir la posición subordinada de las mujeres. Por lo mismo, resulta central el trabajo de reflexión y discusión sobre dichas problemáticas como de hecho lo están haciendo ya las propias organizaciones de mujeres indígenas en varias regiones del país.

Por último quiero reflexionar sobre las implicaciones de una antropología jurídica en los debates políticos sobre el reconocimiento de los derechos indígenas y los derechos de las mujeres. El estudio que hemos realizado tiene la virtud de mostrar, desde las prácticas y las representaciones sociales, la trama de conflictos y poderes que instituyen la relación entre los sexos en las comunidades, así como el papel de las normas en su legitimación. Nos ha permitido asimismo dar cuenta de cambios importantes que están viviendo las comunidades, debido a las dinámicas globales que la impactan y al peso de las organizaciones indígenas y no indígenas en el espacio de las comunidades. En estos procesos se tejen nuevos discursos legales y nuevas formas de mediación entre el Estado y los pueblos indígenas, que revelan tensiones y contradicciones, pero también las direcciones distintas existentes en los procesos sociales. En este sentido el recurrir a la ley del Estado o al discurso de los derechos no necesariamente debe verse como la claudicación al reconocimiento de las jurisdicciones indígenas, sino como opciones discursivas que permitan fortalecerla, siempre que éstas se encuentren en manos de autoridades indígenas y respondan a sus proyectos. En el momento actual me parece que lo que está en juego es cómo defender una visión que al mismo tiempo que reconoce las autonomías indígenas potenciando su desarrollo garantice que dentro de ellas se generen también espacios plurales que permitan construir nuevas propuestas y opciones no autoritarias, sin perder de vista los propios marcos culturales e identitarios de las distintas colectividades.

\section{Notas}

${ }^{1}$ El concepto de sistema sexo-género es desarrollado por Rubin (1996) con el fin de referirse al conjunto de disposiciones por el cual las diferencias sexuales biológicamente marcadas son construidas culturalmente por la intervención humana y social.

${ }^{2} \mathrm{El}$ análisis comparativo que realizamos a continuación no considera el caso de las mujeres indígenas urbanas.

${ }^{3}$ La violación resulta ser efectivamente uno de los delitos más graves y recurrentes contra mujeres indígenas; la mayor parte de ellas suelen quedar impunes ante la falta de pruebas.

${ }^{4}$ Como lo señala la antropología jurídica, las disputas son la punta de iceberg de una serie de conflictos y relaciones sociales previas que revelan el entramado social y cultural que las estructuran (cfr. Van Velsen, 1978).

${ }^{5}$ Soledad González (1999) ha llamado a este proceso el paso del matrimonio concertado al matrimonio romántico. Para profundizar en los cambios sociales y su impacto en las prácticas de noviazgo y matrimoniales en distintos contextos, ver D’Aubeterre (2000).

\section{Bibliografía}

Chenaut, Victoria, 1999, Honor, disputas y usos del derecho entre los totonacas del Distrito Judicial de Papantla, Tesis de Doctorado en Ciencias Sociales. El Colegio de Michoacán, Zamora.

—, (en prensa), "Prácticas jurídicas e interlegalidad, en el distrito judicial de Papantla, Veracruz” en Sierra, María Teresa (editora), "Haciendo justicia": interlegalidad, derecho y género en regiones indígenas, CIESAS, México.

Collier, Jane, 1995, El derecho zinacanteco, CIESAS - UNICACH, México

—, (en prensa), "Cambio y continuidad en los procedimientos legales zinacantecos", en Sierra, María Teresa (editora), "Haciendo justicia”: interlegalidad, derecho y género en regiones indígenas, CIESAS, México.

D’Aubeterre, María Eugenia, 2000, Elpago de la novia, El Colegio de Michoacán/Universidad de Puebla, México.

González, Soledad, 1999, "Las costumbres de matrimonio en el México indígena contemporáneo", en Beatriz Figueroa Campos (coord.), México diverso y desigual: Enfoques Sociodemográficos, sOMEDE / El Colegio de México, México. 
Nicasio, Maribel, 2001, Procuración de justicia e interlegalidad en Metlatónoc, municipio indígena de la montaña de Guerrero, Tesis de maestría en Antropología Social, CIESAS, México. —, (en prensa) "Procuración de justicia e interlegalidad en un municipio indígena de la montaña de Guerrero", Guerrero, México.

Rubin, Gayle, 1996, "El tráfico de mujeres: notas sobre la economía política del sexo" en Martha Lamas (comp.), El género: la construcción cultural de la diferencia sexual, Miguel Porrúa ed., México, pp. 35-97.

Sierra, María Teresa, 1995, "Articulaciones entre la ley y las costumbres: estrategias jurídicas de los nahuas", en Chenaut, Victoria y María Teresa Sierra (coord.)

Pueblos indígenas ante el derecho, CIESAS-CEMCA, México.

—, 2000, "Hay derechos humanos en Zacapoaxtla: legalidad, derecho y género en la Sierra norte de Puebla”, Actas del XII Congreso Internacional de Derecho Consuetudinario y Pluralismo Legal, Universidad de Chile y Universidad de Tarapacá.
-, (en prensa) (editora), "Haciendo justicia": Interlegalidad, derecho y género en regiones indígenas, CIESAS, México.

-, (en prensa), "Interlegalidad, justicia y derechos en la sierra norte de puebla", en Sierra Maria Teresa (editora), "Haciendo justicia”: Interlegalidad, derecho y género en regiones indigenas, CIESAS, México.

—, (en prensa), "Interlegalidad, justicia y derechos en la sierra norte de Puebla", CIESAs, México.

Vallejo, Ivette, 2000, "Mujeres maseualmej y usos de la legalidad: conflictos genéricos en la Sierra norte de Puebla", Tesis de Maestría en Antropología Social, CIESAs, México. Vallejo, Ivette, (en prensa), "Relaciones de género, mujeres nahuas y usos de la legalidad en el municipio de Cuetzalan, Puebla", en Sierra, María Teresa (ed.), "Haciendo justicia": Interlegalidad, derecho y género en regiones indígenas, CIESAS, México. Van Velsen, J. (1967), "The extended case method and situational analysis" en A.L. Epstein (ed.), The craft of social anthropology, G.B. Tavistock Publication. 